\title{
Particles Heat Treating in a Pulsating Gas Stream $\dagger$
}

\author{
A. Parnas \\ American Combustion, Inc., *
}

\begin{abstract}
The subject of the paper is the application of pulsating flow to the particles heat treating. Pulsation influence mechanisms are segregated by the author into two groups: quasi-steady-state (when, at every point in time, an instantaneous value of the heat transfer rate may be calculated with the equations obtained for steady-state conditions) and unsteady - state which includes the remainder. It is shown that, depending on the process parameters, pulsations can increase, not influence, or even reduce heat transfer. Resonance phenomena and large size particles, for which the influence of gravity is substantial, receive special attention. The heat transfer intensification may be achieved due to increases in both heat transfer coefficient and particle residence time. Longitudinal pulsations (when the pulsation and main flow velocities have the same direction) may be preferable in the unsteady-state range of parameters. Transverse pulsations (when the pulsation velocity is perpendicular to the main flow) can be more effective in the quasi-steady-state range. Nonuniformity of heat treating in pulsating flow, influence of the main flow turbulence, trigger effects, and different methods of pulsation generation, including pulsating combustion, are discussed. In conclusion some recommendations are given for designing practical processes.
\end{abstract}

\section{Introduction}

The use of a pulsating flow for intensification of heat and mass transfer in gas suspensions was proposed long ago, and the basics of heat treating particles in subsonic pulsating flows were developed in the period of approximately 1945-1970. Hundreds of papers devoted to the subject have been being published, and sometimes it is not easy to differentiate correct and incorrect, new and well-known ideas. The purpose of this work is not to cover all aspects of the problem (a special monograph is needed for this) but only to discuss its basics. The author's personal opinion is presented, and the list of references is short because only articles which illustrate the problem have been included. The author was involved for many years in investigation of unsteady-state heat transfer, heat transfer in pulsating flow, and pulsating combustion phenomena, and he hopes that the paper will help engineers to better understand the mechanisms of pulsation action and to select the proper range of parameters providing profitable results of pulsation usage.

\footnotetext{
* Norcross, Georgia, USA
}

† Received 15 March, 1996

\section{Basic relationships}

Amount of heat transferred by convection from gas to the particle, can be described with the equation:

$$
q_{p}=\int_{o}^{t} h\left(T_{g}-T_{p}\right) d t,
$$

As it is seen from equation (1), to increase the heat transferred to the particle one should increase heat transfer coefficient, $h$, or/and increase gas temperature, $T_{g}$, or/and increase heat treating time, $t$, (residence time of the particles in the apparatus). The value of $T_{g}$ may be limited with technological requirements: particle material may be sensitive to temperature, etc. Besides, from an economical viewpoint, it is always preferable to use low cost energy, and, therefore, lower gas temperature. For instance, $T_{g}$ cannot exceed the theoretical combustion temperature of a selected fuel. Particle residence time, $t$, is also limited because it is inversely proportional to the average velocity of the particle which cannot be lower than the particle terminal (deposition) velocity. Therefore, increase of a heat transfer coefficient, $h$, is often the only possible method of increasing heat transferred to the particle.

The dimensionless relationship for the heat transfer coefficient can be obtained with the theory of dimensions. Assuming $B i_{p}$ number is small, gas velocity is 
nituch less than the sound speed, and mass transfer process doesn't significantly influence heat transfer, the following relation may be applied:

$$
\begin{aligned}
N u_{p}= & N u_{p}\left(\operatorname{Re}_{p}, G r_{p}, \operatorname{Pr}, S r_{p}, K n_{p}, T_{p} /\right. \\
& \left.T_{g}, \mathcal{E}, L / d, a / d\right)
\end{aligned}
$$

Parameters $\mathcal{E}$ and $L / d$ account for the influence of turbulence. The $\operatorname{Pr}$ number is almost constant for gases and may be excluded from pulsation influence analysis. The $G r_{p}$ number may be also excluded if an influence of gravitational convection is negligible (in the range of $R e_{p}>20$ this is correct if $G r_{p}$ " $\left.R e_{p}{ }^{2}\right)$. $K n_{p}$ number may be excluded for large particles, when $K n_{p} \leqslant 10^{-3}$. For small particles, or in the case of heat transfer at low pressure, when the mean free path of gas molecules is comparable with the dimension defining the heat transfer process (thickness of the boundary layer), a gas temperature jump at the surface of the particle and $K n_{p}$ number should be taken into account. With these considerations, the relationship for heat transfer in pulsating flow becomes:

$$
N u_{p}=N u_{p}\left(R e_{p}, K n_{p}, S r_{p}, T_{p} / T_{g}, \mathcal{E}, L / d, a / d\right)
$$

Two parameters $\left(S r_{p}\right.$ and $\left.a / d\right)$ account for the influence of pulsations in the equation (3). It can be shown that each of them may be replaced with another independent parameter. For instance, the ratio of $v_{a} / V_{m}$ is often used instead of the parameter $a / d$.

\section{Direct and indirect effects}

Pulsations can influence heat transfer "directly" and "indirectly." Indirect effects appear when pulsations work like a trigger initiating significant change of the flow near the particle. In these cases the action of pulsations can be sometimes replaced with the other equivalent steady - state action. For instance, the transition of the laminar boundary layer into turbulent boundary layer at the sphere surface can be initiated both with pulsations and with the Prandtl ring.

Among the conceivable indirect reasons of the mean heat transfer change due to flow pulsations, there are following: initiating of the flow stall (when the steady flow was unstalled), displacement of the flow separation location; transition of the laminar boundary layer into turbulent one, displacement of the transition location; change of the vortex movement pattern behind the particle, etc.

\section{Quasi - steady - state heat transfer}

If an amplitude and frequency of pulsations are not large, and if there are no "trigger" effects, heat transfer is quasi-steady-state. It means that at every point in time an instantaneous value of heat transfer rate may be calculated with the equation obtained for the steady-state conditions:

$$
N u_{p}=N u_{p}\left(R e_{p}, K n_{p}, T_{p} / T_{g}, \mathcal{E}, L / d\right)
$$

where the relative velocity $(V-u)$ is used in $R e_{p}$. Instantaneous value of $V$ must be calculated as a geometrical sum of the average (main), $V_{a v}$, and pulsating, $v$, relative velocities. For the quasi - steady - state case equation (4) is further simplified, and an influence of pulsation is taken into account with one, instead of two, dimensionless parameter containing a pulsating velocity amplitude. Assuming the values of $T_{p} / T_{g}, \mathcal{E}$, and $L / d$ are the same in pulsating and steady state flows, it is easy to obtain the ratio of the heat transfer coefficients:

$$
h / h_{0}=(1 / P) \int_{0}^{p}\left[(V-u) /\left(V_{0}-u_{0}\right)\right]^{n} d t
$$

The values of $V$ and, therefore, of $h / h_{0}$, depend on the direction of pulsating velocity. For definiteness sake assume the pulsating velocity is Sin function of time, and the $n=0.5$. The last value is usually a good approximation for rounded and not very large particles. If the velocity of pulsations is perpendicular to the main velocity $V_{0}$, formula (5) changes to the following one:

$$
\begin{aligned}
h / h_{0}= & (1 / 2 \pi) \int_{0}^{2 \pi}\left\{1+\left[\left(V_{a}-u\right) \operatorname{Sin} x /\right.\right. \\
& \left.\left.\left(V_{0}-u_{0}\right)\right]^{2}\right\}^{0.25} d x
\end{aligned}
$$

If the directions of the pulsating and main velocities are congruent, the influence of pulsations is described with the formula:

$$
\begin{aligned}
h / h_{0}= & (1 / 2 \pi) \int_{0}^{2 \pi} \mid 1+\left(v_{a}-u\right) \operatorname{Sin} x / \\
& \left.\left(\mathrm{V}_{0}-u_{0}\right)\right|^{0.5} d x
\end{aligned}
$$

Values of $h / h_{0}$ calculated with formulas (6) and (7) are presented on Figure 1. It can be seen that perpendicular pulsations (curve 1) substantially increase heat transfer coefficient if a value of an amplitude of relative pulsating velocity is comparable with the main velocity. Longitudinal (congruent) pulsations (curve 2) increase heat transfer only in the range $\left(v_{a}-u\right) /\left(V_{0}-u_{0}\right)>1.8$. 


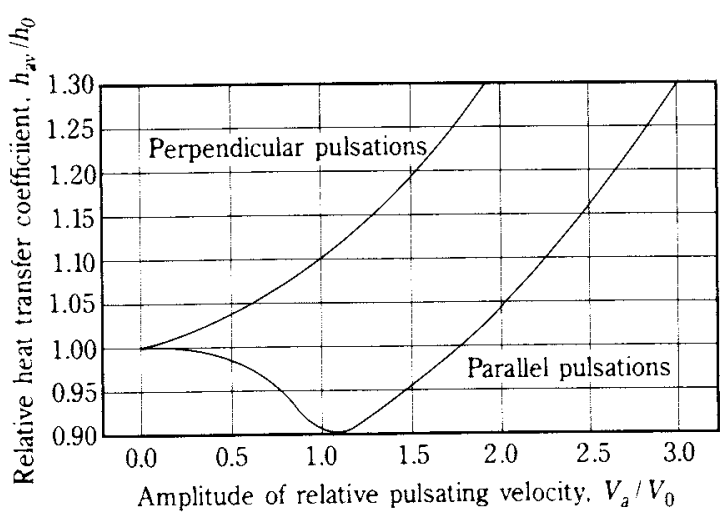

Fig. 1 Influence of pulsations on quasi-steady-state heat transfer coefficient.

To evaluate actual pulsation use benefits, the instantaneous values of relative velocities, and then heat transfer coefficients, residents time, and the average value of transferred heat (formula (1)) must be calculated. There are no universal formulas for such calculations. Usually they must be done numerically with the use of some empirical data. The accuracy of results will depend on a real particles shape and size distribution, parameters of main and pulsating flow, material to gas flow rate ratio, dimensions of apparatus, introduction velocity of particles, and the location of the entrance. Though analytical results may be obtained only with some simplifying assumptions, they proved to be useful as a tool of estimation of the possible benefits of pulsations. Most of the known analytical results are related to the very small ("airborne") particles. In this paper we present some results for the important case of large particles [1]. The following assumptions were made:

1. A constant mass particle is moving vertically upwards entrained by a gas stream oscillating with a period $P$. Gas velocity equals $V_{g}=V_{1}=V_{a v}$ $(1+m)$ during the interval $t_{1}$ of the cycle period, and $V_{g}=0$ during the interval $t_{2}=P-t_{1}$.

2. Gas specific weight is negligibly small in comparison with that of a particle.

3. Collisions of the particle with walls and with other particles are not considered.

4. Drag coefficient of the particle does not depend on the gas velocity and particle orientation.

Expressions for a particle velocity were obtained for the so-called "steady" interval of particle movement (steady in the sense that the particle motion in each successive period does not differ from its motion in the preceding period). Heat transfer then was calculated with a quasi - steady - state assumption that in each point of time the relation $N u \sim \propto\left|V_{g}-u\right|^{\mathrm{n}}$ is valid. As a particle velocity in the upward steady state flow is $\left(V_{0}-V_{t}\right)$ and $N u_{0} \sim \propto V_{t}^{n}$, it is easy to see that

$$
N u_{a v} / N u_{0}=\left[\left(V / V_{t}-u / V_{t}\right)^{n}+m\left(u / V_{t}^{n}\right] /(1+m),\right.
$$

and the ratio of particle residence times is:

$$
t / t_{0}=\left(V_{0}-V_{t}\right) / u \text {. }
$$

Analysis of the obtained solution revealed the minimum mean gas velocity, $V_{\min }$, required to transport particles in a pulsating flow is less than that velocity in a flow without pulsations (which is the terminal settling velocity of the particle, $\left.V_{t}\right)$ :

$$
V_{\text {min }} / V_{t}=(1+m)^{-0.5} \text {. }
$$

This allows substantial reduction of the pulsation gas flow rate and even the use of the regime with $V_{a v} \leq V_{t}$. Due to the possible substantial reduction of the average (mean) gas and particle velocities in a pulsating flow, the gain in the particle residence time may prove to be greater than the possible reduction of the heat transfer coefficient, and then the quantity of the transferred heat may be increased. Heat transfer increase may be also achieved in the opposite case, when the residence time reduces but the coefficient of heat transfer increases. In the work [1] two important cases were analyzed: 1. Equal flow rates $\left(V_{a v}=V_{0}\right)$, and 2. Equal safety margin for reliable pneumatic transportation $\left[V_{a v} / V_{0}\right.$ $\left.=(1+m)^{-0.5}\right]$ in pulsating and steady - state flows. As an illustration, a part of the results of calculations for the case $\left(V_{a v}=V_{0}\right), n=0.8$, and safety ratio $V_{0} / V_{t}=3$ are presented on Figure 2. It is seen that, while retention time reduces, coefficient of heat transfer and heat transferred to the particle in the pulsating flow increase as compared to the flow without pulsations. Maximum value of transferred heat corresponds to $m \approx 2$ and slightly reduces if $m$ increases.

The above examination has been made for the "steady" section of the apparatus. Heat transfer was evaluated also for the so-called accelerating section. For the limiting case, when a particle is introduced in the apparatus with the initial velocity $u=0$, the following formula is valid:

$$
N u_{a v} / N u_{0}=\left(V_{a v} / V_{0}\right)^{n} /(1+m) .
$$

It can be seen from (11) that in the accelerating section heat transfer reduces for both condition $V_{a v}=V_{0}$ 


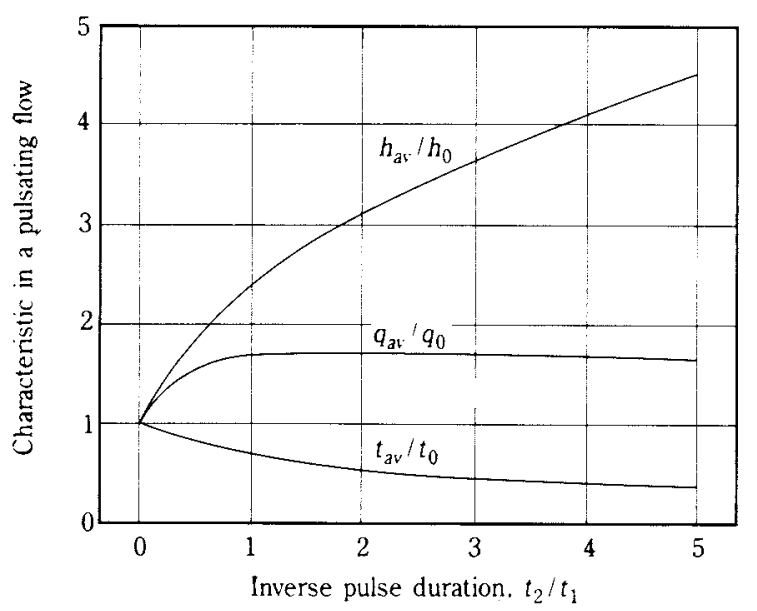

Fig. 2 Residence time and heat transfer in quasi - steady - state upward pulsating flow. $V_{a v}=V_{0}, V_{0} / V_{t}=3$.

and condition $V_{a v} / V_{0}=(1+m)^{-0.5}$. Therefore, the employment of the quasi-steady-state pulsating flow may be not profitable if the apparatus is relatively short and, therefore, the length of accelerating section is substantial.

\section{Unsteady-state effects}

As is evident from the foregoing that the substantial heat transfer increase in quasi - steady - state regime can be achieved only if the magnitudes of pulsation and main velocities are of the same order. Two means to generate large pulsations - a special valve - pulsator installation or/and providing for the resonance pulsations - have certain shortcomings. There were many attempts to intensify a process with the small amplitude pulsations, for instance, by applying sound or ultrasound waves to the gas flow. While some results were promising, the other didn't show a change of heat transfer at all. It is obvious that the knowledge of the "critical" pulsation parameters which divide steady-state and unsteady-state pulsation regimes, can help to explain the unsuccessful results and to correctly select pulsation parameters.

Unsteady-state effects may appear when the time of gas parameters change is small, and, therefore, steady-state heat transfer is not achieved. Physically, it can be explained by the well-known phenomena: creation of the hydrodynamic and temperature boundary layers, as well as of the vortex zone behind the body, takes time. The magnitude of this time depends mainly on the shape of the body and on the numbers $R e_{p}$ and $\operatorname{Pr}$. To experimentally define this time, transient heat transfer of the cylinder with the vortex zone behind it was investigated in the work [2]. A cylinder was selected as a typical bluff body and, therefore, the results of the tests could be applied, at least semi-quantitatively, to other shape bluff bodies (spheres, etc.). In that work parameters of the gas flow changed abruptly, and the time of transition to the steady - state heat transfer regime was measured. The results proved that the transition time may be approximately estimated with the dimensionless number $\mathrm{Ho}_{0} \approx 5$. This value is in a good agreement with the frequency of developing and separation vortexes which in the broad range of $R e$ numbers is defined with the relationship:

$$
S r_{p} \approx 0.2
$$

To obtain values of critical pulsation parameters, the usual theoretical approach can be used: the steady - state range of hydrodynamic and related to them heat transfer parameters is achieved if the local acceleration member in the Navier - Stokes equation is small in comparison to convective acceleration members [3, etc.]:

$$
\partial V_{g} / \partial t \ll V_{g} \partial V_{g} / \partial L
$$

In the case of periodic pulsations the analysis was fulfilled in the work [5]. It can be assumed: $\partial V_{g} / \partial t \sim$ $V_{g} / P, \partial V_{g} / \partial L \sim V_{g} / d$. Then the equation (13) transforms into an expression:

$$
S r_{p} \ll 1
$$

The condition (14) can be refined for the particular cases of pulsations perpendicular and parallel to the main flow velocity. For harmonic pulsations parallel to the main flow, the relative gas velocity is $\left[V_{0}+v_{a}\right.$ $\operatorname{Sin}(\omega t)-u]$. If particles are very small, the relative pulsation velocity $\left[v_{a} \operatorname{Sin}(\omega t)-u\right]$. is small, and the situation around the particle is quasi - steady - state. The maximum influence of pulsations may be achieved for the large particles, when $u \approx 0$. Then $\partial V_{g} / \partial t \sim$ $v_{a} / P$, and expression (14) can be replaced with an inequality

$$
\operatorname{Sr}\left(v_{a} / V_{0}\right) \ll 1 .
$$

The other form of the same quasi - steady - state condition can be obtained if to take into account equation $v_{a}=2 \pi$ a f:

$$
S_{p}(a / d)^{0.5} \ll 1 .
$$


Pf the direction of pulsations is perpendicular to the main velocity, the absolute value of relative gas velocity for the large particles is $\left[V_{0}^{2}+v_{a}^{2} \operatorname{Sin}^{2}(\omega t)\right]^{0.5}$. If the ratio $v_{a} / V_{0}$ is small, the influence of velocity direction pulsation on the heat transfer is negligible. As $\partial V_{g} / \partial t \sim v_{a}{ }^{2} / V_{0} P$, the quasi-steady - state condition for perpendicular pulsations is:

$$
S_{p}\left(v_{a} / V_{0}\right)^{2} \ll 1 \text {. }
$$

or in the other form:

$$
\operatorname{Sr}_{p}(a / d)^{2 / 3} \text { «1. }
$$

To experimentally verify relations (17) and (18) and define magnitudes of critical parameters, heat transfer between air flow and a vibrating small size cylinder was investigated in the work [4]. The use of body vibrations, instead of gas pulsations, allowed to accurately measure pulsation parameters: velocity and amplitude. The experiments confirmed the structure of the given above critical parameters complexes. Their values for longitudinal pulsations were defined as follows (it was assumed that deviation from quasisteady - state heat transfer should be $1 \%$ ):

or

$$
\left[\operatorname{Sr}_{p}(a / d)^{0.5}\right]=0.03 \sim 0.055,
$$

$$
\left[\operatorname{Sr}_{p}\left(v_{a} / V_{0}\right)\right]=0.008 \sim 0.019 .
$$

Expressions (19) and (20) were obtained in the ranges: $R e_{p}=200 \sim 3000, S r_{p}<0.12, v / V_{0}<0.6$. The turbulence influence wasn't expressly analyzed though parameter $\varepsilon$ was measured with a thermoanemometer. The value of $\varepsilon$ depended on the flow rate and varied in the range $2 \sim 6 \%$. Parameter $\mathrm{L} / \mathrm{d}$ in the tests was constant. Smaller values in formulas (19) and (20) correspond to the smaller turbulence of the air flow. It can be explained thus: the less are "natural" pulsations of the main flow, the greater is an influence of additional disturbances caused by induced pulsations. The results, probably, may be used in the extended range of $R e_{p}$ numbers if the pattern of the gas flow is similar to the investigated one.

Critical parameters for perpendicular pulsations were larger than critical parameters for parallel vibrations. This is in a good agreement with the fact that the acceleration of perpendicular pulsations is less than the acceleration of parallel pulsations of the same amplitude and frequency: it can be shown that their ratio equals $0.5 v_{\mathfrak{a}} / V_{0}$. The exact values of critical parameters for perpendicular pulsations were not defined because experiments in the work [4] were conducted at the values of $S r_{p}$ number close to 0.2 , and the maximum of heat transfer related to the vortex separation [5] masked the "direct" unsteady-state-effect.

\section{Related phenomena}

\subsection{Resonance}

Three different resonance phenomena may exist in chambers and ducts filled with a two-phase gas particles media:

1. Local "gas-particle" resonance, corresponding to the value of $S r_{p} \approx 0.2$, when a frequency of induced pulsations is close to the frequency of vortexes separation from the particle surface [5]. As the well-developed vortex flow can appear only behind the large particles, when $R e_{p} \geq 40 \sim$ 50 , and because the values of $S r_{p}$ and $R e_{p}$ numbers for different size particles may differ many times in the real polydisperse flows, the possibility of the substantial influence of this kind of resonance is, probably, smail.

2. Local "gas-wall" resonance, when a frequency of induced pulsations is close to the frequency of vortexes developing in the gas-wall boundary layer. This resonance influences mainly heat transfer in the area close to the wall and between the wall and gas - particle flow.

3. Large scale acoustic resonance, when a frequency of induced pulsations is close to the one of natural acoustic frequencies of the gas-particle media in the chamber and the ducts. Some experimental investigations [5, etc.] proved that the amplitudes of the resonance pulsations may be very large, even greater than the mean stream velocity. In this case the instantaneous gas velocity vector at certain times can be directed opposite to the mean velocity at the antinodes of the standing waves. Calculations showed [6] that the large amplitude resonance pulsations could substantially increase heat transfer both in the "steady" and accelerating sections of the apparatus.

\subsection{Turbulence of the main flow}

The gas flow turbulence can be treated as "natural" pulsations having a wide range of amplitudes and frequencies. As indicated above, the less are turbulent disturbances of the main flow, the greater is an influence of the induced pulsations. In practical systems an average amplitude of turbulent pulsations usually vary in the range of $2 \sim 6 \%$ of the main flow velocity. To noticeably increase heat transfer (if there are no "indirect" effects) the induced velocity pulsations 
should be larger than existing turbulence velocities.

\subsection{Nonuniformity of heat treating}

It is known that small particles, as a rule, are heated faster than the larger ones in a steady - state poly-disperse particle - gas flow. Pulsations can add to this nonuniformity. It can be shown that, if the small and the large particles follow the same hydrodynamic force law, the possible relative increase of heat transferred to the small particles is larger than that to the large particles. Nevertheless, if the goal is to increase heat transfer to the large particles, and overheating of small particles is allowable, the use of pulsations may be justified. Pulsations may also reduce the nonuniformity of heat treating if the difference of particle sizes is so big that their movement is defined by different relations between the hydrodynamic force and relative velocity.

An important cause of nonuniform heat treating can be continuous charge of material in the apparatus with the low frequency pulsation flow. As the particles are introduced in different phases of pulsation cycle, they receive different initial velocities, and their residence times and transferred heats are different. The differences can be especially large if particle introduction velocities are directed opposite to the main stream flow [1].

\subsection{Agglomeration and separation}

The increased difference by pulsations in small and large particle velocities, sometimes even reverse movement of a part of particles in some phases of pulsation cycle, increases both a local material concentration and the probability of agglomeration. The possible increase of residence times increases the probability of larger particles (or agglomerated clusters) fall out and separation.

\section{Basic types of pulsation generators}

\subsection{Pulse valve-interrupter}

A simple generator of pulsations is a valve - interrupter of the gas flow. It is usually installed in the gas supply line and made, for instance, as a rotating disk or cylinder with holes. An amplitude of pulsations depends on the size of holes, and the frequency is defined by both the number of the holes and the frequency of rotation. An additional pressure loss and an influence of pulsations on the accuracy of the flow rate measurements are inherent in such pulsators.

\subsection{Sound generator}

Sound waves in gases are longitudinal relatively to the source of the sound. The amplitude of sound waves velocity depends mainly on the sound intensity, I, and the gas properties: composition, density, and temperature. For the air at atmospheric pressure and temperature $20^{\circ} \mathrm{C}$, an amplitude of pulsation velocity is: $v_{a}=0.007 \mathrm{~m} / \mathrm{sec}$ at $\mathrm{I}=100$ $\mathrm{db}, 0.069 \mathrm{~m} / \mathrm{sec}$ at $120 \mathrm{db}, 0.69 \mathrm{~m} / \mathrm{sec}$ at $140 \mathrm{db}$, and $6.93 \mathrm{~m} / \mathrm{sec}$ at $160 \mathrm{db}$. The known experimental investigations showed that usually only big amplitude sound waves (I> 130 140 db) could increase the forced convection heat transfer rate. To protect the maintenance personnel against the high intensity sound, the apparatus must be well insulated.

\subsection{Pulsating combustion}

The period of $15-20$ years after The World War II was the time when a lot of engineers and scientists investigated pulsating combustion. They tried to develop the new technology of efficient gas and liquid fuel combustion, and to use the high temperature pulsating flow of combustion products for heating, drying and other industrial processes. However, the optimistic expectations, based initially on the German missile pulsating combustion engines experience, were not realized. Additional stresses in structural elements caused by pulsations which are especially large in the case of resonances can substantially reduce the structure endurance limit. Combustion chambers, as well as other parts and structures influenced by pulsations, must be reinforced, and, therefore, their weight and cost increased. This is probably the main reason why pulsating combustion is not used now not only in transport engines, industrial and power plants but, probably, in all types of large scale apparatus. Like in the case of other pulsating generators, the decision of pulsating combustion chamber use should be done in each particular case after complete evaluation of the possible expenses and benefits.

\subsection{Periodic change of the apparatus cross section area}

Pulsations of gas velocity may be organized along the particle path by the periodical change of the channel cross section. Particles decelerate in a large area section of apparatus and accelerate in the small area section. In this case there is no need in a special pulsation generator use. An analysis of such process with vertical transportation of large particles was done in the work [7]. 
transfer coefficients and residence times of the particles. After pulsation parameters are defined, pulsation generator and other equipment providing for these parameters may be selected. The final decision can be made after a comparison of all benefits and expenses related to the pulsation use. The expenses depend on the additional pressure loss, possible quality reduction due to secondary effects (agglomeration, etc.), and additional capital investment including the noise suppression and unit structure reinforcement, if necessary.

\section{Nomenclature}

\subsection{Expenditure of energy}

The use of pulsations usually causes an additional energy consumption. Even if the energy consumption by the pulsation generator itself is small, the possible additional hydraulic pressure losses in the transportation system may be substantial. As a rule, a relative increase of heat transfer coefficient is less than the relative increase of pressure loss. Like in the case of the capital investment, the actual energy consumption depends on the specific type and parameters of the unit and process.

\section{Conclusion}

Though there is no simple method to accurately predict the result of each particular pulsation application, available data allows one to explain the mechanism of pulsation influence, to define the desirable range of pulsation parameters, to estimate anticipated benefits and expenses, and to select the proper type of pulsation generator and other equipment. The following procedure may be recommended for designing a pulsation process. First of all, absolute and relative particle velocities and interphase heat transfer coefficients in the main stream without pulsations should be calculated. The knowledge of the main stream turbulence characteristics is a plus. Then one must evaluate the boundary parameters between quasi-steady-state and unsteady state heat transfer regimes for the particles to be treated. If the quasi-steady-state heat transfer regime is anticipated the transverse pulsations (when the pulsation velocity is perpendicular to the main flow) are usually preferable. Longitudinal pulsations (when the pulsation and main flow velocities have the same direction) may be preferable in the unsteady-state range of parameters. The possibility of trigger effects, large scale and local resonance phenomena should be verified. The total effect of pulsation application can be obtained by calculation of the product of the heat

a Pulsation (vibration) amplitude, $\mathrm{mm}$

d Particle diameter, $\mathrm{mm}$

$f \quad$ Pulsation (vibration) frequency, $\quad \mathrm{Hz}$

$h \quad$ Heat transfer coefficient, $\quad \mathrm{W} / \mathrm{m}^{2} /{ }^{\circ} \mathrm{C}$

$I \quad$ Sound intensity $\mathrm{db}$

$L$ Scale of turbulence, m

$m=t_{2} / t_{1}$-cycle parameter dimensionless

$n \quad$ Exponent in the relation $\mathrm{Nu} \sim \mathrm{Re}^{\mathrm{n}}$

$\begin{array}{llr}q & \text { Heat } & \mathrm{J} / \mathrm{m}^{2} \\ P & \text { Period of pulsation (vibration) } & \mathrm{sec} \\ t & \text { Time } & \mathrm{sec} \\ T & \text { Temperature } & { }^{\circ} \mathrm{C} \\ V & \text { Pulsation (vibration) velocity } & \mathrm{m} / \mathrm{sec} \\ u & \text { Particle velocity } & \mathrm{m} / \mathrm{sec} \\ V & \text { Gas velocity } & \mathrm{m} / \mathrm{sec} \\ X & =2 \pi \mathrm{ft} \text { - variable of integration dimensionless } \\ \varepsilon & \text { Intensity of turbulence dimensionless, } \% \\ \lambda & \text { Thermal conductivity } & \mathrm{W} / \mathrm{m} /{ }^{\circ} \mathrm{C} \\ g & \text { Acceleration of gravity, } & \mathrm{m} / \mathrm{sec}^{2} \\ \nu & \text { Kinematic viscosity of gas } & \mathrm{m}^{2} / \mathrm{sec}^{2} \\ l & \text { Mean free path of gas molecules, } & \mathrm{mm}\end{array}$

\section{Dimensionless Numbers:}

$B i_{p}$ Biot number, $\quad \mathrm{hd} / \lambda_{\mathrm{p}}$

$G r_{p}$ Grashof number, $\quad \mathrm{gd}^{3}\left(\mathrm{t}_{\mathrm{g}}-\mathrm{t}_{\mathrm{p}}\right) /\left(\mathrm{t}_{\mathrm{g}}+273\right) / \mathrm{v}_{\mathrm{g}}{ }^{2}$

Ho Dimensionless time number, $\quad \mathrm{tV} / \mathrm{d}$

$K n_{p}$, Knudsen Number $\quad 1 / d$

$\mathrm{Nu}_{p}$ Nusselt number $\quad \mathrm{hd} / \lambda_{\mathrm{g}}$

$\operatorname{Pr}$ Prandtl number $\quad \nu_{\mathrm{g}} c_{\mathrm{g}} e_{\mathrm{g}} / \lambda_{\mathrm{g}}$

$R e_{p}$ Reynolds number

$\mathrm{Sr}_{p}$ Strouhal number

$\mathrm{Vd} / \mathrm{v}_{\mathrm{g}}$
$\mathrm{fd} / \mathrm{V}$

\section{Subscripts:}

a Amplitude value

av Average (mean value)

$g$ Gas

$\min$ Minimum value 
$a^{N}$ Value in the steady - state flow

p Particle

$t$ Terminal velocity

\section{References}

1) Parnas, A.L., and S.S. Zabrodskiy: Collected articles "Heat and Mass Transfer in Disperse Media", 79-86, Belarus Academy of Sciences, Institute of Heat and Mass Transfer, Nauka i Tekhnika, Minsk, USSR, 1965 (in Russian)

2) Parnas, A.L.: Collected articles "Problems of Unsteady-State Heat and Mass Transfer", 30-35, Belarus Academy of Sciences, Institute of Heat and Mass Transfer, Nauka i Tekhnika, Minsk, USSR, 1965 (in Russian)

3) H. Schlichting: Grenzschicht-Theorie, Verlag G. Braun-Karlsruhe, Germany, 1958.

4) Parnas, A.L.: Collected articles "Problems of Unsteady-State Heat and Mass Transfer', 25-29, Belarus Academy of Sciences, Institute of Heat and Mass Transfer, Nauka i Tekhnika, Minsk, USSR, 1965 (in Russian).

5) Parnas, A.L.: Journal of Engineering Physics, 10, 37 - 44, 1964, Int. Chem. Eng., 213 -217, 2, 1967.

6) Zabrodskiy, S.S., and A.L. Parnas: Journal of Engineering Physics, 6, 778-782, 1965.

7) Elperin, I.T., and A.L. Parnas: Journal of Engineering Physics, 3, 352-356, 1966.

\section{Author's short biography}

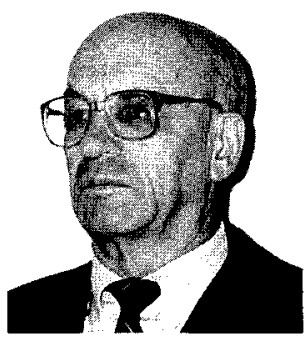

\section{Anatole Parnas}

Dr. Anatole Parnas graduated from Moscow Power Institute and Luikov Institute of Heat and Mass Transfer of Belarus Academy of Sciences. His major research interests are related with applications of Theory of Combustion, Thermal/Fluid Science, and Mechanics to various industrial processes: particulate materials heat treating, heat treating of metals, heat transfer and thermal stresses in electronic devices. He also fulfilled some experimental research, analytical and numerical analysis of theoretical problems of heat transfer and mechanics, including unsteady-state convective heat transfer, heat transfer in dispersed media, pulsating combustion, and vibrations and stresses in shafts and plates. Currently he is with American Combustion, Inc. 